\title{
First bulk and surface results for the ATLAS ITk Strip stereo annulus sensors
}

\author{
S.H. Abidi ${ }^{1}$, A.A. Affolder ${ }^{k}$, J. Bohm ${ }^{\mathrm{j}}$, J. Botte ${ }^{\mathrm{a}}$, B. Ciungul ${ }^{1}$, K. Dette ${ }^{\mathrm{l}}$, Z. Dolezali ${ }^{\mathrm{i}}$, C. Escobar ${ }^{\mathrm{g}}$, V. Fadeyev $^{\mathrm{k}}$, \\ J. Fernandez-Tejero ${ }^{\text {b }}$, C. García Argos ${ }^{\mathrm{e}}$, D. Gillberg ${ }^{\mathrm{a}}$, K. Hara ${ }^{\mathrm{m}}$, M. Hauser ${ }^{\mathrm{e}}$, R.F.H. Hunter ${ }^{\mathrm{a}}$, K. Jakobs ${ }^{\mathrm{e}}$, J.S. Keller ${ }^{\mathrm{a}}$, \\ P. Kodys ${ }^{\mathrm{i}}$, T. Koffas ${ }^{\mathrm{a}}$, Z. Kotek ${ }^{\mathrm{j}}$, J. Krolli ${ }^{\mathrm{j}}$, C. Lacasta ${ }^{\mathrm{g}}$, V. Latonova ${ }^{\mathrm{j}}$, J. Loenker ${ }^{\mathrm{d}}$, D. Madaffari ${ }^{\mathrm{g}}$, M. Mikestikova ${ }^{\mathrm{j}}$, \\ M. Miñano ${ }^{g}$, S. Y. Ng ${ }^{\mathrm{f}}$, R.S. Orr ${ }^{1}$, U. Parzefall ${ }^{\mathrm{e}}$, D. Rodriguez ${ }^{\mathrm{g}}$, U. Soldevila ${ }^{\mathrm{g}}$, J. Stastny $^{\mathrm{j}}$, M. Stegler ${ }^{\mathrm{c}}$, J. Suzuki ${ }^{\mathrm{m}}$,

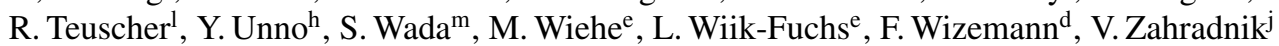 \\ ${ }^{a}$ Physics Department, Carleton University, 1125 Colonel By Drive, Ottawa, Ontario, K1S 5B6, Canada \\ ${ }^{b}$ Centro Nacional de Microelectronica (IMB-CNM, CSIC), Campus UAB-Bellaterra, 08193 Barcelona, Spain \\ ${ }^{c}$ Deutsches Elektronen-Synchrotron, Notkestraße 85, 22607 Hamburg, Germany \\ ${ }^{d}$ Experimentelle Physik IV, TU Dortmund, Dortmund, Germany \\ ${ }^{e}$ Physikalisches Institut, Albert-Ludwigs-Universität Freiburg, Hermann-Herder-Str. 3, 79104 Freiburg-im-Breisgau, Germany \\ ${ }^{f}$ Institut für Physik, Humboldt-Universität zu Berlin, Newtonstraße, Berlin, Germany \\ ${ }^{g}$ Instituto de Física Corpuscular (IFIC) - CSIC-University of Valencia, Parque Científico, C/Catedrático José Beltrán 2, E-46980 Paterna, Spain \\ ${ }^{h}$ IPNS, KEK, 1-1 Oho, Tsukuba, Ibaraki 305-0801, Japan \\ ${ }^{i}$ Faculty of Mathematics and Physics, Charles University, V Holesovickach 2, Prague, CZ18000 The Czech Republic \\ ${ }^{j}$ Academy of Sciences of the Czech Republic, Institute of Physics, Na Slovance 2, 18221 Prague 8, Czech Republic \\ ${ }^{k}$ Santa Cruz Institute for Particle Physics (SCIPP), University of California, Santa Cruz, CA 95064, USA \\ ${ }^{l}$ Department of Physics, University of Toronto, 60 Saint George St., Toronto M5S 1A7, Ontario, Canada \\ ${ }^{m}$ Institue of Pure and Applied Sciences, University of Tsukuba, 1-1-1 Tennodai, Tsukuba Ibaraki 305-8571, Japan
}

\begin{abstract}
A novel microstrip sensor geometry, the stereo annulus, has been developed for use in the end-cap of the ATLAS experiment's strip tracker upgrade at the HL-LHC. Its first implementation is in the ATLAS12EC sensors, a largearea, radiation-hard, single-sided, AC-coupled, $\mathrm{n}^{+}$-in-p design produced by the ITk Strip Sensor Collaboration and fabricated by Hamamatsu Photonics in early 2017. The results of the initial testing of two ATLAS12EC batches are presented here with a comparison to specification. The potential of the new sensor shape to reinvigorate endcap strip tracking is explained and its effects on sensor performance are isolated by comparing the bulk mechanical and electrical properties of the new sensor to the previous iteration of prototype, the conventional barrel ATLAS12A sensor. The surface properties of the new sensor are evaluated for full-size unirradiated sensors as well as for mini sensors unirradiated and irradiated with protons up to a fluence of $2.2 \times 10^{15} \mathrm{n}_{\mathrm{eq}} \mathrm{cm}^{-2}$. The results show that the new stereo annulus ATLAS12EC sensors exhibit excellent performance and the expected irradiation evolution.
\end{abstract}

Keywords: HL-LHC, ATLAS ITk, $\mathrm{n}^{+}$-in-p microstrip sensor, Stereo annulus, Bulk properties, Surface properties, Surface irradiation

\section{Introduction and the Stereo Annulus Geometry}

In the mid-2020s the Large Hadron Colider (LHC) will be upgraded into a High-Luminosity (HL-LHC) phase which will allow significant increases in the instantaneous and integrated luminosity targets of the experiment, up to $7.5 \times 10^{34} \mathrm{~cm}^{-2} \mathrm{~s}^{-1}$ and $4 \mathrm{ab}^{-1}$, respectively. Occupancy, readout, and radiation damage are the main concerns for the detectors which sit poised to capture the order of magnitude more premier physics data than was originally planned for the LHC. For the general purpose ATLAS detector [1] the more intense collision environment requires the complete replace-

Preprint submitted to Elsevier ment of the innermost tracking component to be able to operate for the full high luminosity phase.

The Inner Tracker (ITk) is the all-silicon replacement to the current system. It utilizes two detection geometries; strips and pixels, the granularity and layout of which have been optimized iteratively through extensive data-driven simulation [2]. The strip portion of the ITk, called the ITk Strip Detector, fills the majority of the volume; it is the full $6 \mathrm{~m}$ long extent of the inner cavity and extends from a radius of about $30 \mathrm{~cm}$ to the inner surface of the calorimeter assembly at a radius of $1 \mathrm{~m}$. The Strip Detector layout is conventional for a collider experiment; cylindrically symmetric with a four layer

February 16, 2018 
barrel in the central region $(|z| \leq 1.4 \mathrm{~m})$ and two endcaps each comprised of six discs filling the remaining volume on either end. The discs are made of 32 identical double-sided 'petals' (annular segments) each with nine sensors per side in six designs.

The ITk Strip Detector will utilize an entirely new sensor geometry, the stereo annulus, which possesses several advantages over the conventional configuration. In detectors like ATLAS the magnetic field is aligned along the beam pipe causing charged particle trajectories to bend in the transverse plane. In the endcaps the strips are oriented radially to allow the most sensitive feature on the sensors, the strip pitch, to capture the most significant dimension, the bending angle. To avoid an insufficient resolution in the radial distance from the beam pipe (obtained by centimeter long strips), the strips are rotated by a small degree, called the stereo angle. In this manner the strips from the sensors on the two sides of a disc make a diamond shaped grid allowing a much more precise measurement.

Conventionally these tracking requirements are filled by taking a symmetric trapezoidal wedge shaped sensor and rotating it while placing it on the support structure. This is the approach used in the current ATLAS SCT. Since the sensors are mounted on circular annular discs the straight tangential edges of the conventional approach are suboptimal leaving incomplete coverage at the radial extrema of the disc and requiring unequal strip lengths even in a single sensor segment. Furthermore the rotated placement of the sensors leads to increased logistical load as they must be distributed in manners that are congruent with strict hermeticity and radiation length requirements. All these issues are solved when moving to the aptly named stereo annulus design. The radial edges and the strips on the surface have been rotated by the stereo angle while the tangential edges have been replaced by circular arc segments concentric on the beam pipe center. From a geometrical standpoint this design is optimized for the annular disc endcap environment yielding the best coverage and channel uniformity with the most efficient stereo angle implementation.

In this document the initial test results for the firstever stereo annulus sensor fabrication are discussed. These are the ATLAS12EC a third iteration of novel, large-area, radiation-hard sensor designed by the ITk Strip Sensor Working Group and the first endcap prototype. Produced in early 2017 by Hamamatsu Photonics, Ref. [3], the design originated in 2012 and is similar to the contemporary barrel design; the ATLAS12 fabricated in 2014 [4]. This kinship makes a comparison of the bulk character of the ATLAS12 and ATLAS12EC perfect for isolating the effect of the stereo annulus ge- ometry on sensor performance.

The $4 \mathrm{ab}^{-1}$ integrated luminosity target involves unprecedented particle fluences resulting in $\sim 1.6 \times$ $10^{15} \mathrm{n}_{\mathrm{eq}} \mathrm{cm}^{-2}$ non-ionizing energy loss (NIEL) and $\sim 67$ Mrad total ionizing dose (TID) in the ITk Strip Detector volume (includes 1.5 safety factor). Surface as well as bulk damage is expected [5, 6, 7, , 8, 9]. In this document the surface characteristics of the ATLAS12EC wafers before and after proton irradiation are contrasted while Ref. [10] contains the bulk irradiated results.

\section{Test Samples and Irradiation}

The ITk Strip sensor prototypes use a radiation-hard AC-coupled, $\mathrm{n}^{+}$-in-p design. This allows the costeffective use of single-sided sensors that are resistant to bulk leakage current increase and that can still operate effectively under partial depletion [4]. The one major disadvantage of the $\mathrm{n}^{+}$-in-p material, that of strip isolation, is overcome with $\mathrm{p}^{+}$traces (p-stops) whose doping level and single, common, symmetric geometry have been optimized iteratively [11, 12]. Also implemented are optimized punch through protection (PTP) structures, see Refs. [12, 4, 8], which protect the coupling dielectric in the event of large charge liberation in the bulk (in the case of beam splash).

The ATLAS12EC has a large surface area of $90.0 \mathrm{~cm}^{2}$ in a design which mimics the sensor closest to the beam pipe (the Ring- 0 or R0). The annular edges of this stereo annulus sensor are obtained with 16 flat cuts each. A $20 \mathrm{mrad}$ stereo angle is implemented in four strip segments of constant lengths and angular pitch. The lengths ranging from $19 \mathrm{~mm}$ to $32 \mathrm{~mm}$ have been chosen for occupancy, radiation-hardness, and readout considerations. The angular pitch of $193 \mu \mathrm{rad}$ in the inner two segments and $172 \mu \mathrm{rad}$ in the outer two segments produce pitch variations of $73.5 \mu \mathrm{rad}$ to $83.9 \mu \mathrm{rad}$ across the sensor surface allowing 4360 strips to be arranged in manners compatible with the readout electronics assembly. Float-zone, $\left\langle\begin{array}{lll}1 & 0 & 0\end{array}\right\rangle$ p-type silicon wafers approximately $320 \mu \mathrm{m}$ thick, are used. Further details of the ATLAS12EC design are presented in Ref. [13].

Roughly 60 ATLAS12EC sensors from two batches are examined for their bulk character. The results are compared to their conventionally shaped kin, the ATLAS12A, consisting of 120 sensors (Ref. [14]). They are comprised of 5128 strips arranged with a $74.5 \mu \mathrm{m}$ pitch in four segments $23.9 \mathrm{~mm}$ in length within a bias ring area of $91.8 \mathrm{~cm}^{2}$. 
The ATLAS12EC full-size sensors are accompanied by $1 \mathrm{~cm} \times 1 \mathrm{~cm}$ mini-sensors. They come in several varieties with variant pitch. Three values of $70 \mu \mathrm{m}, 75 \mu \mathrm{m}$ and $80 \mu \mathrm{m}$ (denoted narrow, default, and wide respectively) are used to approximate the range on the full size sensors. In Section 4 the surface results of 20 minis, unirradiated and irradiated to NIEL fluences of $0.5,1.1$ and $2.2 \times 10^{15} \mathrm{n}_{\mathrm{eq}} \mathrm{cm}^{-2}$ are presented along with the surface characterization of a few unirradiated full-size sensors. The irradiation is carried out with $70 \mathrm{MeV}$ protons from the CYRIC ${ }^{1}$ facility with all samples annealed for $80 \mathrm{~min}$ at $60^{\circ} \mathrm{C}$ to the minimum of the annealing curve. For these AC-coupled sensors, surface damage comes from ionizing interactions whereas a CYRIC fluence of $1.0 \times 10^{15} \mathrm{n}_{\mathrm{eq}} \mathrm{cm}^{-2}$ corresponds to a TID in silicon of $\sim 80 \mathrm{Mrad}[8]$. These interactions can seed trapping sites in the oxide volume and at the $\mathrm{SiO}_{2}-\mathrm{Si}$ interface. The relative $\mathrm{e}^{-}$and $\mathrm{h}^{+}$mobilities in $\mathrm{SiO}_{2}$ as well as the field configuration of the $\mathrm{n}^{+}$-in-p type device leads to the buildup of positive space charge in the oxide volume and in particular near the silicon interface [5, 7]. This as well as the increase in trapping sites at the interface leads to greater charge exchange and a reinforced inversion layer which has consequences for the sensor operation such as reduced channel isolation and increased surface leakage current [12, 8].

The ATLAS12EC full-size and mini sensors have been tested at 12 institutes. All sensors are tested in cleanroom conditions of $20^{\circ} \mathrm{C}$ to $30^{\circ} \mathrm{C}$ and relative humidity of $10 \%$ to $40 \%$. Dry storage $(<5 \%$ relative humidity) is used between tests. The irradiated devices have been tested cold $\left(-20^{\circ} \mathrm{C}\right.$ to $\left.-30{ }^{\circ} \mathrm{C}\right)$ to avoid further annealing.

\section{Bulk Sensor Characteristics}

\subsection{Mechanical Properties}

The ATLAS12EC technical specifications place a number of restrictions on the mechanical character of the sensors, among these are the sensor flatness $(<200 \mu \mathrm{m}$ unstressed), thickness $((310 \pm 25) \mu \mathrm{m})$, and edge quality (no chips or cracks extending $50 \mu \mathrm{m}$ or more inwards). The last of these is a visual inspection of the sensor under magnification, which all sensors passed. While the first two quantities are obtained with non-contact (optical) measurement techniques with accuracy of a few micron $(\leq 5 \mu \mathrm{m})$ in all three dimensions.

The sensor flatness (or bow) measurements are obtained in a grid of 100-130 points across the surface.

${ }^{1}$ Cyclotron and Radioisotope Center, Tokohu University, Japan
The data is analyzed and corrected to remove the tilt of the freely sitting sensor. The flatness or bow is extracted as the difference between the highest and lowest points on the sensor. The obtained profiles for 40 measured sensors follow the dome-like expectation (higher in the center of the wafer). The average bow and its RMS error are $51.18 \mu \mathrm{m}$ and $9.30 \mu \mathrm{m}$ respectively. These values are well within the specification and compare quite closely with a similarly measured sample of 100 ATLAS12A (51.72 $\mu \mathrm{m}$ and $12.36 \mu \mathrm{m})$ [14].

The thickness of the sensors is measured in a minimum of five locations on the sensor. Measurement of a few sensors at the test sites have yielded thicknesses in the range $320 \mu \mathrm{m}$ to $325 \mu \mathrm{m}$, values which are safely within specification and that closely match the supplier's results.

\subsection{Depletion Capacitance}

The bulk capacitance of the sensors is obtained as a function of reverse bias voltage to determine the full depletion voltage, $V_{F D}$, of the sensors. An LCR meter (such as the Wayne Kerr 6440B) in R-C series mode is used to apply the AC probe voltage at a frequency of $1 \mathrm{kHz}$. This frequency is chosen as it lies on the flat part of the bulk capacitance versus frequency curve as measured for the ATLAS12 [14].

The CV curves of 49 ATLAS12EC sensors are shown in Fig. 11. The inverse square of the bulk capacitance is plotted on the $y$-scale to allow easy extraction of the full depletion voltage. Extremely high consistency is obtained in the 49 sensors with the outlier points at high voltages attributable to probe contact issues. The histogrammed results for the extracted $V_{F D}$ are shown in Fig. 2 for 58 sensors, with an average of $303.04 \mathrm{~V}$ and an RMSE of $4.17 \mathrm{~V}$. The nine sensor difference arises from a parasitic capacitance at one site which affected the capacitance values but not the full depletion voltage.

Taking the bias ring enclosed area as the active area and assuming a parallel plate capacitor model allows one to obtain estimates of the active thickness and bulk resistivity from the measured $V_{F D}$ and capacitance at full depletion [4]. The active thickness calculated was $302.3 \mu \mathrm{m}$ or $93 \%$ of the full thickness, a value that agrees qualitatively with the edge-TCT measurements in Ref. [10]. The calculated bulk resistivity was $(3.24 \pm 0.03) \mathrm{k} \Omega \mathrm{cm}$ which meets the specification of $\geq 3.0 \mathrm{k} \Omega \mathrm{cm}$.

Although the ATLAS12 have a different wafer resistivity $(\sim 2.5 \mathrm{k} \Omega \mathrm{cm})$ a meaningful comparison can still be made by looking at the RMSE of the $V_{F D}$ and the active thickness percentages respectively. The ATLAS12 $V_{F D}$ obtained had a RMSE of $8.61 \mathrm{~V}$ for a sample of 
98 sensors [14]. The lower RMSE of the ATLAS12EC indicates a better processing consistency as could be expected from its later fabrication. The active thickness calculated for the ATLAS12 ( 94\%) is in very close agreement with the ATLAS12EC indicating similar high quality processing of the backside implant.

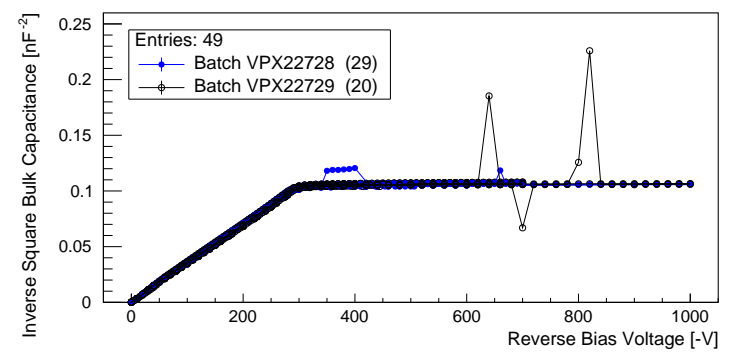

Fig. 1: Inverse square bulk capacitance versus reverse bias voltage for 49 ATLAS12EC sensors. The outlying point on the high voltage plateau can be attributed to poor contact.

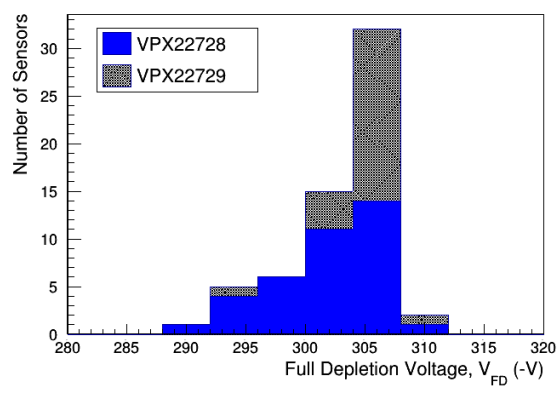

Fig. 2: Histogram of the ATLAS12EC $V_{F D}$. An average of $303.04 \mathrm{~V}$ and an RMSE of $4.17 \mathrm{~V}$ is obtained for 49 sensors.

\subsection{Reverse Bias Leakage Current}

The reverse bias leakage current has been obtained as a function of voltage to ensure that it is stable and within specification. Data is obtained with pico-amp precision source measurement units (SMUs) such as the Keithley 2410 with the reverse bias voltage ramp in steps of $10 \mathrm{~V}$ from $0 \mathrm{~V}$ to $1 \mathrm{kV}$. A $10 \mathrm{~s}$ settle period is allowed at each step before the measurement is taken. For the ATLAS12EC the specifications of a leakage current less than $2 \mu \mathrm{A} \mathrm{cm}^{-2}$ at $20^{\circ} \mathrm{C}$ and $600 \mathrm{~V}$ as well as stability up to $600 \mathrm{~V}$ are used.

The leakage current curve for 59 ATLAS12EC sensors is shown in Fig. 3. Here the current has been normalized by the bias ring area and to $20^{\circ} \mathrm{C}$ using the well-established temperature dependence, Ref. [15]. A minority of sensors showing early breakdown required 'training' in which they were ramped much more slowly before a normal measurement was made. The plot shows that the majority of sensors exhibited ideal IV character showing a stable current plateau and a kink at the full depletion voltage attributable to surface generated currents as the depletion front encompasses the backplane contact [4]. A small number of sensors show signs of instability. An average of $1.95 \mathrm{nA} \mathrm{cm}^{-2}$ with an RMSE of $1.87 \mathrm{nA} \mathrm{cm}^{-2}$ is obtained. This is three orders of magnitude within specification. It also compares to an average of $14.72 \mathrm{nA} \mathrm{cm}^{-2}$ with RMSE of $59.35 \mathrm{nA} \mathrm{cm}^{-2}$ obtained for 118 ATLAS 12 [14].

The levels of instability in the ATLAS12EC are very reasonable when compared to the ATLAS12. For the ATLAS12EC $7 \%$ and $15 \%$ show this feature at voltages below $600 \mathrm{~V}$ and $1000 \mathrm{~V}$ reverse bias respectively. For the 118 conventionally shaped ATLAS12 the values are $14 \%$ and $28 \%$ respectively, proving that the instability is not related to the stereo annulus shape. In fact a number of results have been gathered which support the hypothesis that passivation issues are the culprit, the main evidence being the humidity sensitivity of the IV character. Observations such as the inverse relations between microdischarge onset and long term testing instability versus ambient humidity are obtained for both generations of sensor and are the subject of further study.

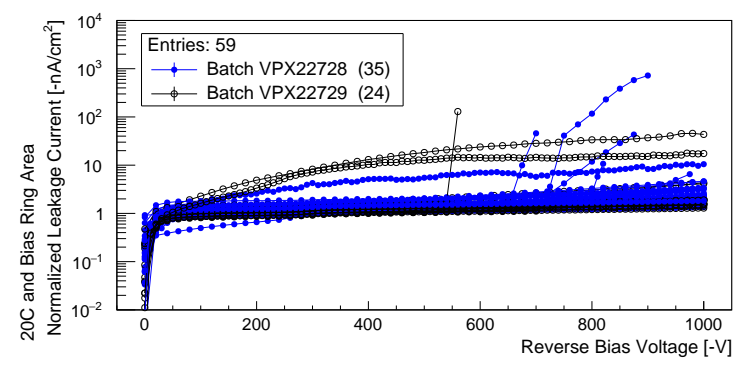

Fig. 3: Reverse bias leakage currents as a function of reverse bias voltage for 59 ATLAS12EC sensors, normalized by the bias ring enclosed area and to $20^{\circ} \mathrm{C}$.

\section{Sensor Surface Properties}

\subsection{Strip Coupling Capacitance}

The coupling capacitance, $C_{\text {coup }}$, is the capacitance between the strips and the implants. It must be large to allow the signal to be extracted efficiently and consistent for optimal channel response. A specification of $\geq 20 \mathrm{pF} \mathrm{cm}^{-1}$ at $1 \mathrm{kHz}$ is set. Measurements of the coupling capacitance have been conducted on a full strip segment of 1026 strips with a results of $(25.21 \pm$ 
0.02) $\mathrm{pF} \mathrm{cm}^{-1}$ as well as for a few strips across the sensor surface for a small number of sensors with all results falling in the range $24.89 \mathrm{pF} \mathrm{cm}^{-1}$ to $28.37 \mathrm{pF} \mathrm{cm}^{-1}$. Measurements of $C_{\text {coup }}$ have also been conducted on the irradiated minis with negligble change after proton irradiation up to the maximal fluence observed.

\subsection{Bias Resistance}

The resistive nature, $R_{\text {bias }}$, of the polysilicon traces connecting the implants to the bias rail is measured to ensure that the value is large enough for effective bias dispersion and isolation of the channels. High consistency is also required in this feature to avoid any transverse field gradients in the bulk. As such $(1.5 \pm 0.5) \mathrm{M} \Omega$ is specified for the ATLAS12EC design. Like the coupling capacitance this value has been measured for all 1026 strips of a single segment as well as for a number of strips across the surface of a few other full-size sensors. Here the results are $(1.59 \pm 0.01) \mathrm{M} \Omega$ for the segment and $1.47 \mathrm{M} \Omega$ to $1.52 \mathrm{M} \Omega$ for the other tests with a flat bias voltage dependency. For irradiated mini sensors a small positive fluence dependency as well as a negative temperature dependency were observed, matching expectations [12, 8]. The essentially flat dependency versus reverse bias voltage is maintained.

\subsection{Interstrip Resistance}

The interstrip resistance, $R_{\text {int }}$, is the resistance between neighbouring $\mathrm{n}^{+}$implants. For effective DC isolation of the channels through the silicon bulk $R_{i n t}$ it must be much larger than $R_{\text {bias }}$. A specification of $>10 \times R_{\text {bias }}$ at a strip region depleting bias voltage of $300 \mathrm{~V}$ is applied. $R_{\text {int }}$ is measured by contacting three neighbouring implants and applying a small test voltage (up to $\pm 5 \mathrm{~V}$ in $1 \mathrm{~V}$ steps) to the outer ones while reading the induced current at the center from the voltage across its bias resistor. High impedance, high isolation devices such as the Keithley 6517 and 2000 are used. A small number of strips on a few full-size sensors have been measured with all results residing above $10 \mathrm{G} \Omega$. For irradiated minis, a decrease in the interstrip resistance with fluence is expected from the oxide's positive space charge and interface trap build-up as well as, less importantly, from NIEL damage to the bulk [12, 8]. This decrease is observed in conjunction with a positive reverse bias voltage dependency which is also expected (the inversion layer shrinks as bias voltage increases). A flat bias voltage evolution is observed in all unirradiated sensors. The strip isolation specification is met at $\sim 50 \mathrm{~V}$ for the highest fluence tested indicating more than sufficient performance of the optimized p-stops implemented in the ATLAS12EC $\mathrm{n}^{+}$-in-p design. The temperature dependence of $R_{\text {int }}$ is understood (Ref. [8]) and is used to normalize the $400 \mathrm{~V}$ reverse bias point to $-20^{\circ} \mathrm{C}$ for comparison to previous sensor generations. The updated plot of Fig. 10 from Ref. [8] is shown in Fig. 4 where the ATLAS12EC find good agreement.

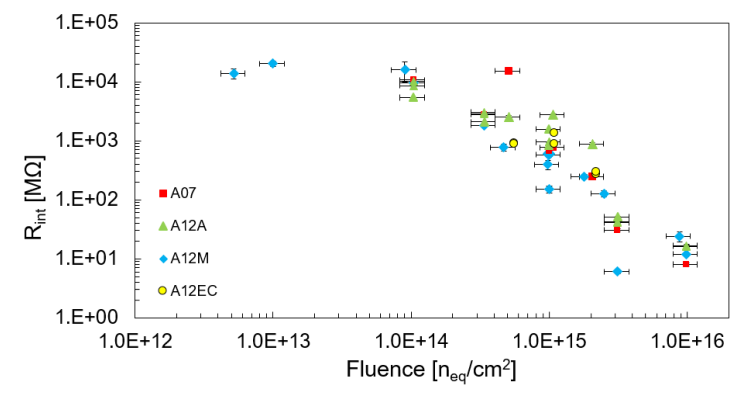

Fig. 4: Interstrip resistance, $R_{\text {int }}$, evolution with proton fluence at $400 \mathrm{~V}$ reverse bias and normalized to $-20^{\circ} \mathrm{C}$. Comparison to previous prototype iterations, the ATLAS07 and ATLAS12, is made.

\subsection{Interstrip Capacitance}

The interstrip capacitance, $C_{\text {int }}$, is the capacitance between neighbouring top metal strips. In the $\mathrm{AC}$ design it is the dominant source of noise at the chargesensitive front-end readout chip and must be minimized. A value of $<0.9 \mathrm{pF} \mathrm{cm}^{-1}$ at $300 \mathrm{~V}$ and $100 \mathrm{kHz}$ is specified. From a few strips measured on a small number of sensors values of $C_{i n t}$ ranging from $0.61 \mathrm{pF} \mathrm{cm}^{-1}$ to $0.89 \mathrm{pF} \mathrm{cm}^{-1}$ have been obtained. The interstrip capacitance dependency on reverse bias voltage at $100 \mathrm{kHz}$ is shown in Fig. 5 for at least one strip from each segment of a full-size sensor. The variant peak at very low bias voltage is attributable to the variable surface state at the start of measurement (dependent upon sensor bias and humidity history). Matching expectations (see Ref. [8]), the measurements of the mini sensors depict an almost flat bias voltage evolution of $C_{i n t}$ for the irradiated devices saturated at the pre-irradiated full depletion value.

\subsection{Punch Through Protection}

In the event of miscontrolled beam during HL-LHC activity large charge liberation in the bulk may result in the voltage on the implants increasing up to half the bias [12]. With the breakdown voltage of the coupling capacitor dielectric layer specified as $100 \mathrm{~V}$ the use of the PTP structures which allow a controlled soft breakdown between implant and bias rail is necessary. The PTP effectiveness is quantified by the onset voltage of breakdown which should be much smaller than $100 \mathrm{~V}$, 


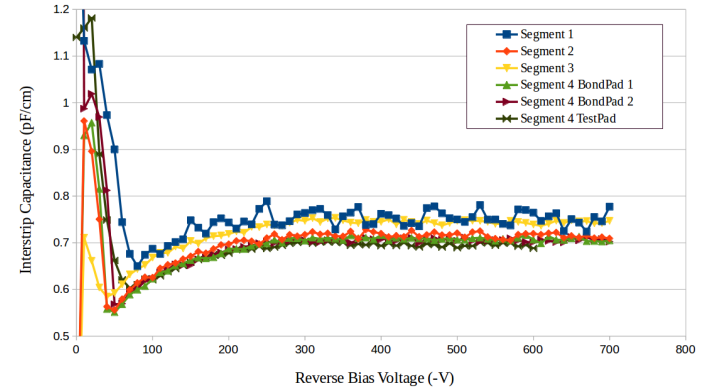

Fig. 5: Interstrip capacitance evolution as a function of bias voltage at a frequency of $100 \mathrm{kHz}$ for at least one strip from each segment of a full-size sensor (Test and Bond pad indicate segment ends).

the rate of decrease of the gap's effective resistance after breakdown which should be high, and the current flow at $100 \mathrm{~V}$ which should be maximal. These values are measured directly between implant and rail. A typical onset voltage of less than $20 \mathrm{~V}$ with a steep decrease in the effective resistance and a large current flow even at $40 \mathrm{~V}$ are obtained for the unirradiated sensors. The PTP was also measured for the proton irradiated minis. The largest effect is a moderation in the decrease rate of the effective resistance after breakdown. For the highest fluence point the effective resistance still falls below $0.2 \mathrm{M} \Omega$ before $30 \mathrm{~V}$ indicating excellent PTP performance.

\section{Summary and Conclusions}

The initial results for the bulk and surface character of the ATLAS12EC $n^{+}$-in-p sensor have been reported along with the surface property of proton irradiated minis. The sensors showed excellent consistency in both bulk and surface quality with all structures performing as expected and within specification. The ATLAS12EC sensors are unique as they are the first to ever use the stereo annulus geometry a feature which has been shown to have considerable advantage over the conventional design. Comparison to the more conventionally designed ATLAS12 sensors showed that no deleterious effects on sensor operation were introduced by the optimized stereo annulus geometry.

\section{Acknowledgements}

The research was supported and financed in part by Canada Foundation for Innovation, the National Science and Engineering Research Council (NSERC) of Canada under the Research and Technology Instrumentation (RTI) grant SAPEQ-2016-00015; the Ministry of Education, Youth and Sports of the Czech Republic coming from the project LM2015058 - Research infrastructure for experiments at CERN; the Federal Ministry of Education and Research, BMBF, Germany; USA Department of Energy, Grant DE-SC0010107; the Spanish Ministry of Economy and Competitiveness through the Particle Physics National Program, ref. FPA2015-65652-C4-4-R (MINECO/FEDER, UE), and co-financed with FEDER funds.

\section{References}

[1] The ATLAS Collaboration, The ATLAS Experiment at the CERN Large Hadron Collider Journal of Instrumentation 3 (08) (2008) S08003.

URL http://stacks.iop.org $/ 1748-0221 / 3 / i=08 / a=$ S08003

[2] ATLAS Collaboration, ATLAS Inner Tracker Strip Detector: Technical Design Report, Tech. Rep. ATL-TDR-025 / LHCC2017-005.

[3] Hamamatsu Photonics, http://www .hamamatsu.com

[4] Y. Unno et al., Development of $n+-$ in - p large-area silicon microstrip sensors for very high radiation environments - ATLAS12 design and initial results, Nuclear Instruments and Methods in Physics Research A 765 (2014) 80-90.

[5] T.R. Oldham and F.B. McLean, Total Ionizing Dose Effects in MOS Oxides and Devices, IEEE Transactions on Nuclear Science 503 (2003) 483-499.

[6] I. Pintilie et al., Radiation-induced point- and cluster-related defects with strong impact on damage properties of silicon detectors, Nuclear Instruments and Methods in Physics Research A 611 (2009) 52-68.

[7] T. Poehlsen et al., Charge losses in segmented silicon sensors at the $\mathrm{Si}-\mathrm{SiO} 2$ Interface, Nuclear Instruments and Methods in Physics Research A 700 (2013) 22-29.

[8] M. Mikestikova et al., Study of surface properties of ATLAS12 strip sensors and their radiation resistance, Nuclear Instruments and Methods in Physics Research A 831 (2016) 197-206.

[9] K. Hara et al., Charge collection and field profile studies of heavily irradiated strip sensors for the ATLAS inner tracker upgrade, Nuclear Instruments and Methods in Physics Research A 831 (2016) 1-8.

[10] V. Cindro et al., Measurement of charge collection in irradiated miniature sensors for the upgrade of ATLAS Phase-II Strip tracker, this issue.

[11] Y. Unno, Development of radiation-tolerant silicon microstrip sensor for the ATLAS inner tracker at the SLHC, Nuclear Instruments and Methods in Physics Research A 623 (2010) 165167.

[12] S. Lindgren et al., Testing of surface properties pre-rad and postrad of n-in-p silicon sensors for very high radiation environment, Nuclear Instruments and Methods in Physics Research A 636 (2011) S111-S117.

[13] C. Lacasta et al., Design of the First Full-Size ATLAS ITk Strip Sensor for the Endcap Region, this issue.

[14] L.B.A. Hommels et al., Detailed studies of full-size ATLAS12 sensors, Nuclear Instruments and Methods in Physics Research A 831 (2016) 167-173.

[15] A. Chilingarov, Temperature dependence of the current generated in the Si bulk, Journal of Instrumentation 810 (2013) P10003. 